\title{
Origin of the first Hund rule and the structure of Fermi holes in two-dimensional He-like atoms and two-electron quantum dots
}

\author{
Tokuei Sako ${ }^{1}$, Josef Paldus ${ }^{2}$, Atsushi Ichimura ${ }^{3}$, and Geerd \\ H.F. Diercksen ${ }^{4}$ \\ ${ }^{1}$ Laboratory of Physics, College of Science and Technology, Nihon University, 7-24-1 \\ Narashinodai, Funabashi, 274-8501 Chiba, Japan \\ ${ }^{2}$ Department of Applied Mathematics, University of Waterloo, Waterloo, Ontario \\ N2L 3G1, Canada \\ ${ }^{3}$ Institute of Space and Astronautical Science, JAXA, Sagamihara 229-8510, Japan \\ ${ }^{4}$ Max-Planck-Institut für Astrophysik, Karl-Schwarzschild-Strasse 1, D-85741 \\ Garching, Germany \\ E-mail: ${ }^{1}$ sako@phys.ge.cst.nihon-u.ac.jp, ${ }^{2}$ paldus@uwaterloo.ca ${ }^{3}$ \\ ichimura@isas.jaxa.jp ${ }^{4}$ ghd@mpa-garching.mpg.de
}

\begin{abstract}
The lowest singlet-triplet pair of states of the two-electron twodimensional quantum dots and the corresponding pair of states of the two-dimensional helium-like systems have been studied by the full configuration interaction method focusing on the origin of the first Hund rule. The one- and two-electron components of the singlet-triplet energy gap show distinct trends for the systems studied in the regime of small nuclear charge $Z_{\mathrm{n}}$ or of small confinement strength $\omega$. The $\left(0 \sigma_{g}\right)\left(1 \pi_{u}\right)$ singlet state in quantum dots is characterized by a larger electron repulsion than its counterpart triplet state for all values of $\omega$ while this relationship gets inverted for the corresponding $(1 s)(2 p)$ singlet-triplet pair of He-like systems for small values of $Z_{\mathrm{n}}$, such as $Z_{\mathrm{n}}=2$ or 3 . The internal part of the full configuration interaction wave functions has been extracted and visualised in the three-dimensional internal space $\left(r_{1}, r_{2}, \phi_{-}\right)$to rationalize the observed trends. The singlet probability density of Helike systems located originally near the Fermi holes is shown to migrate into regions where either $r_{1}$ or $r_{2}$ are large while the corresponding singlet probability of quantum dots stays close to the Fermi holes. Their differences and their observed trends are rationalized on the basis of the structure of the genuine and conjugate Fermi holes.
\end{abstract}

PACS numbers: 31.10.+z, 31.15.A-, 32.10.-f

Submitted to: J. Phys. B: At. Mol. Phys.

\section{Introduction}

The origin of Hund's first multiplicity rule concerning the spin multiplicity $[1,2,3,4,5$, $6,7,8]$ has been a source of ongoing theoretical studies since the beginning of quantum 
mechanics until today $[9,10,11,12,13,14,15,16,17,18,19,20,21,22,23,24,25,26,27]$ (see Ref. [27] for an excellent summary of relevant studies prior to 2010). A significant part of earlier efforts focused on singly-excited states of helium and of helium-like ions representing the simplest examples to which Hund's multiplicity rule applies: A given orbital configuration $(1 s)(n l)$ where $n$ and $l$ denote the principle and the angular momentum quantum numbers, respectively, gives rise to only one singlet-triplet pair of states and according to Hund's rule the triplet state is always lower in energy than the singlet state [see Ref. [28] for cases of double-excitation configurations, such as $(2 p)(2 p)$, $(2 p)(3 d)$, etc., that involve more than one singlet-triplet pair].

Even for this simplest example the reasons for the triplet state to have a lower energy than the corresponding singlet state are far from being straightforward. One of the major puzzling features is the fact that the triplet state of the helium atom has a larger electron repulsion energy than the corresponding singlet state $[10,11]$, in contrast to the traditional interpretation based on Slater's paper of 1929 [9]. This implies that contrary to the traditional interpretation the reason for the triplet to have a lower energy is not due to a smaller electron repulsion but must be ascribed to a more compact electron density distribution in the triplet state relative to the singlet state. This then results in a much larger energy decrease due to the nuclear attraction potential that compensates the energy increase due to the electron repulsion $[29,18,19,20,12,14,15,16,17]$. This surprising observation has given rise to another interesting and more fundamental question, namely, why the triplet state has a more compact electron density distribution than the corresponding singlet state in the first place. To rationalize the compactness of triplet electrons Boyd introduced the well-known reduced nuclear screening model $[24,20]$ involving a significant angular interelectronic correlation. Recently, however, this model has been challenged by Sajeev et al. [30] who showed that such an angular correlation that would be essential for an electron in an outer orbital in the triplet state to feel a larger effective nuclear charge and to come closer to the nucleus than the electrons in the singlet state is insignificant. This controversial situation has been recently unraveled by the introduction of a new concept, namely that of the so called conjugate Fermi hole [31].

Our earlier study [32] also investigated the origin of Hund's multiplicity rule in twoelectron artificial atoms or quantum dots. Although the confining potential in these systems is not of Coulomb-type as in natural atoms but more like that of a harmonic oscillator [33] their energy levels are known to obey Hund's multiplicity rule as well $[34,35,30,32]$. Furthermore, due to the harmonic nature of the confining potential the Hamiltonian of quantum dots can be separated into the center-of-mass and the internal degrees of freedom. This, together with a (quasi) two-dimensional nature of these systems, allows a detailed analysis of the internal wave functions that fully accounts for the correlation between the two electrons. It has been shown $[34,31]$ that similarly as in the case of the helium atom the electron density distribution in the triplet state is indeed more compact than that in the corresponding singlet state yet, in contrast to the helium case, the electron repulsion energy of the triplet state never exceeds that of 
the corresponding singlet state.

Motivated by these earlier studies on He-like systems and quantum dots, the present study focuses on the origin of their differences and clarifies the mechanism why for quantum dots the electron repulsion energy is always smaller in the triplet state than in the corresponding singlet state while this relationship is reversed in the He-like systems with small nuclear charge $Z_{\mathrm{n}}$. In this regard it is the concept of genuine and conjugate Fermi holes as well as their structure in the internal space that represents the key to an understanding of the above mentioned differences.

\section{THEORETICAL MODEL AND COMPUTATIONAL METHOD}

In the present study the spatial degrees of freedom of each of the two electrons in both the helium atom and in the quantum dot are confined to a two-dimensional $x y$ plane. Our previous study showed that this two-dimensional helium model reproduces all the characteristic features of the energy spectrum of the real 3D helium atom [31]. By reducing the dimensionality and thus the number of the degrees of freedom the internal part of the wave functions can be easily visualised. This allows an unambiguous manifestation of the origin of the Hund rule.

The electronic Hamiltonian for the two-dimensional helium-like systems and that for the two-electron two-dimensional quantum dot have the following form, respectively,

$$
\begin{aligned}
& \mathcal{H}_{Z}=-\frac{1}{2} \sum_{i=1}^{2} \nabla_{i}^{2}-\sum_{i=1}^{2} \frac{Z_{\mathrm{n}}}{\left|\overrightarrow{r_{i}}\right|}+\frac{1}{\left|\vec{r}_{1}-\vec{r}_{2}\right|}, \\
& \mathcal{H}_{\omega}=-\frac{1}{2} \sum_{i=1}^{2} \nabla_{i}^{2}+\sum_{i=1}^{2} \frac{1}{2} \omega^{2}\left|\vec{r}_{i}\right|^{2}+\frac{1}{\left|\vec{r}_{1}-\vec{r}_{2}\right|},
\end{aligned}
$$

where $\vec{r}_{i}=\left(x_{i}, y_{i}\right)\left[=r_{i}\left(\cos \phi_{i}, \sin \phi_{i}\right)\right]$ for $i=1,2$, while $Z_{\mathrm{n}}$ and $\omega$ represent the nuclear charge of the helium-like systems and the strength of confinement of the quantum dot. The one-electron part of both Hamiltonians has an analytical solution which yields the following eigenenergies

$$
\begin{aligned}
& E_{Z}^{0}=\sum_{i=1}^{2} \frac{-Z_{\mathrm{n}}^{2}}{2\left(n_{i}+\frac{1}{2}\right)^{2}}, \\
& E_{\omega}^{0}=\sum_{i=1}^{2} \omega\left(v_{i}+\frac{1}{2}\right),
\end{aligned}
$$

with $n_{i}$ and $v_{i}(i=1,2)$ denoting the principal and the harmonic-oscillator quatum numbers. Therefore, the energies of the Hamiltonians (1) and (2) are scaled by $Z_{\mathrm{n}}^{2}$ and $\omega$. By introducing the $Z_{\mathrm{n}^{-}}$and $\omega$-scaled coordinates $\vec{s}_{i} \equiv Z_{\mathrm{n}} \vec{r}_{i}$ and $\vec{t}_{i} \equiv \sqrt{\omega} \vec{r}_{i}(i=1,2)$ for the He-like and the quantum dot systems the Hamiltonians (1) and (2) take the form

$$
\mathcal{H}_{Z} / Z_{\mathrm{n}}^{2}=-\frac{1}{2} \sum_{i=1}^{2} \nabla_{s, i}^{2}-\sum_{i=1}^{2} \frac{1}{\left|\vec{s}_{i}\right|}+\frac{1}{Z_{\mathrm{n}}\left|\vec{s}_{1}-\vec{s}_{2}\right|},
$$


Table 1. Parameters for the atomic basis set of two-dimensional helium-like systems in $Z_{\mathrm{n}}$-scaled coordinates.

$$
\begin{array}{rrrr}
\cline { 2 - 4 } & \multicolumn{1}{l}{m_{l}} & \zeta_{\max , l} & \zeta_{\min , l} \\
\hline 0 & 20 & 800.0 & 0.001 \\
1 & 10 & 3.0 & 0.001 \\
2 & 7 & 1.0 & 0.003 \\
\hline & \\
\mathcal{H}_{\omega} / \omega=-\frac{1}{2} \sum_{i=1}^{2} \nabla_{t, i}^{2}+\sum_{i=1}^{2} \frac{1}{2}\left|\overrightarrow{t_{i}}\right|^{2}+\frac{1}{\sqrt{\omega}\left|\overrightarrow{t_{1}}-\overrightarrow{t_{2}}\right|}
\end{array}
$$

These equations show that in contrast to the two-electron potential, the one-electron part of the scaled Hamiltonians (5) and (6) does not depend on $Z_{\mathrm{n}}$ and $\omega$, implying that the effect of the electron-electron interaction on the eigenenergies and wave functions for different $Z_{\mathrm{n}}$ and $\omega$ can be clearly seen using these scaled coordinates.

Two-dimensional Cartesian Gaussian-type functions of the form

$$
\chi^{\vec{a}, \zeta}(\vec{r})=x^{a_{x}} y^{a_{y}} \exp \left[-\zeta\left(x^{2}+y^{2}\right)\right]
$$

have been used to expand the one-electron orbitals for the scaled Hamiltonians (5) and (6). Following the quantum chemical convention these functions are classified as $s^{-}, p-$, $d$-type, etc. for $l=a_{x}+a_{y}=0,1,2$, etc., respectively. A $[20 s 10 p 7 d]$ basis set has been used for He-like systems while a $[1 s 1 p 1 d 1 f 1 g 1 h 1 i]$ basis set with large angular momentum functions has been used for quantum dots [36, 37, 38, 39, 40].

The exponents of the Gaussian functions for the He-like systems have been generated by relying on the geometrical formula [41, 42] (see also Ref. [43] for further information)

$$
\zeta_{j, l}=\alpha_{l} \beta_{l}^{j-1}, \quad j=1,2, \cdots, m_{l} .
$$

The minimum and maximum exponents, $\zeta_{\min , l}$ and $\zeta_{\max , l}$, and the number of components $m_{l}$ for each $l$-shell are listed in Table 1 . In the case of quantum dots all exponents have been chosen as one half of the strength of confinement $[36,37,38]$, i.e., $\zeta=0.5$ in the present case, since the strength of confinement for a harmonic oscillator in the $\omega$-scaled Hamiltonian (6) is unity.

The eigenfunctions and the corresponding energies for the relevant states have been obtained by diagonalising the full configuration interaction (FCI) Hamiltonian matrix. The eigenenergies of the unscaled original Hamiltonians of Eqs. (1) and (2) can then be obtained by multiplying the calculated ernergies by $Z_{\mathrm{n}}^{2}$ and $\omega$, respectively. It should be noted that the singlet-triplet energy gap for the singly-excited states of He-like systems can be produced already at the Hartree-Fock level [10, 11] suggesting that the electron correlation is not essential for engendering Hund's rule for these systems. However, we have chosen FCI here since, apart from its higher reliability relative to the Hartree-Fock 
method, the electron correlation is much more important in the case of quantum dots as well as, generally, for two-dimensional systems relative to thee-dimensional systems.

The internal space for the two-dimensional two-electron systems with circular symmetry can be described by two radial coordinates and an interelectron angle, i.e. $s_{i}$ $\equiv\left|\vec{s}_{i}\right|(i=1,2)$ and $\phi_{-} \equiv\left(\phi_{1}-\phi_{2}\right) / 2$ for He-like systems and $t_{i} \equiv\left|\vec{t}_{i}\right|(i=1,2)$ and $\phi_{-}$ for quantum dots. The domains for these coordinates are $0 \leq s_{i}, t_{i}<\infty(i=1,2)$ and $-\pi \leq \phi_{-} \leq \pi$. The probability densities in each of these internal spaces are obtained from the FCI wave functions by integrating out the coordinate of the complementary angle $\phi_{+} \equiv\left(\phi_{1}+\phi_{2}\right) / 2$ that is associated with the total orbital angular momentum $L$. This two-body correlation function in the internal space is called hereafter the internal wave functions for simplicity. The details of this procedure may be found in a previous paper [31].

\section{Results and discussion}

\subsection{Singlet-triplet energy differences}

The lowest singlet-triplet pair of states of the two-dimensional two-electron quantum dot to which Hund's multiplicity rule applies is the ${ }^{1} \Pi_{u^{-}}{ }^{3} \Pi_{u}$ pair of states [32]. This Hund's pair has the primary configuration $\left(0 \sigma_{g}\right)\left(1 \pi_{u}\right)$ where the number in front of the orbital symmetry label represents the number of nodes of the orbital. For example, the $0 \sigma_{g}$ orbital has no node while the $1 \pi_{u}$ orbital has one angular node corresponding to its angular momentum $l=1$. Thus this pair of states of the quantum dot corresponds to the $(1 s)(2 p)$ singlet-triplet pair of the He-like systems (we note that in a rigorous sense the orbitals of two-dimensional He-like systems should be labeled by the $D_{\infty, \mathrm{h}}$ point group. However, for the sake of convenience we employ the conventional labeling for the He-like systems in order to relate the present results to the corresponding 3D systems). It may also be interesting to study the lowest $\Sigma$ singlet-triplet pair of states of quantum dots that corresponds to the $(1 s)(2 s)$ pair of the He-like systems. However, the lowest singlet $\Sigma$ state has a mixed character involving a singly-excited configuration $\left(0 \sigma_{g}\right)\left(2 \sigma_{g}\right)$ and a doubly-excited configuration $\left(1 \pi_{u}\right)\left(1 \pi_{u}\right)$. This happens in quantum dots since the zero-order energies for these two configurations exactly coincide with each other due to the harmonic nature of the confining potential. Therefore, it is difficult to make a clear comparison for this state between He-like systems and quantum dots where the singlyexcited states of the He-like systems are dominated purely by a single configuration of $(1 s)(n l)$. Therefore, the following analysis focuses mainly on the ${ }^{1} \Pi_{u^{-}}{ }^{3} \Pi_{u}$ and ${ }^{1} P_{-}{ }^{3} P$ pairs of states.

In our previous work [32, 31] the singlet-triplet energy gap for these Hund pairs as well as its partitioning into the one-electron and two-electron components have been examined for different $Z_{\mathrm{n}}$ and $\omega$ values, respectively. The results that are extended by the present calculations to larger $Z_{\mathrm{n}}$ and smaller $\omega$ values are summarised in Fig. 1 where the difference in the total, one-electron and two-electron energies between the 


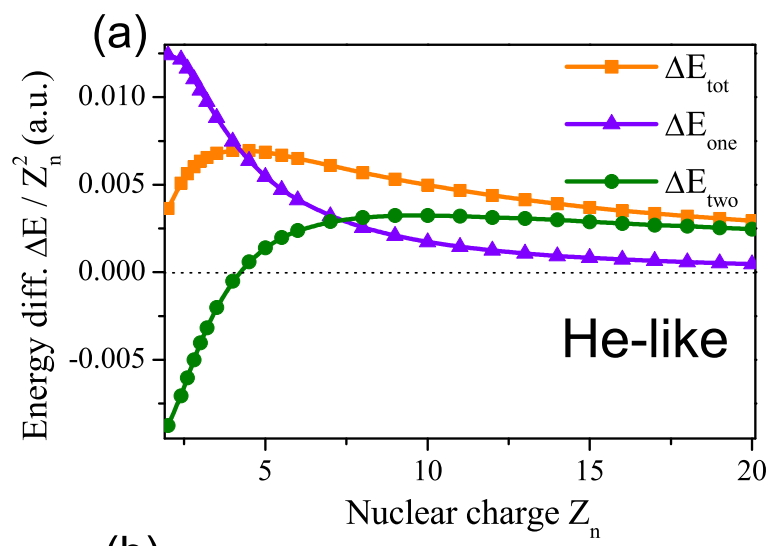

(b)

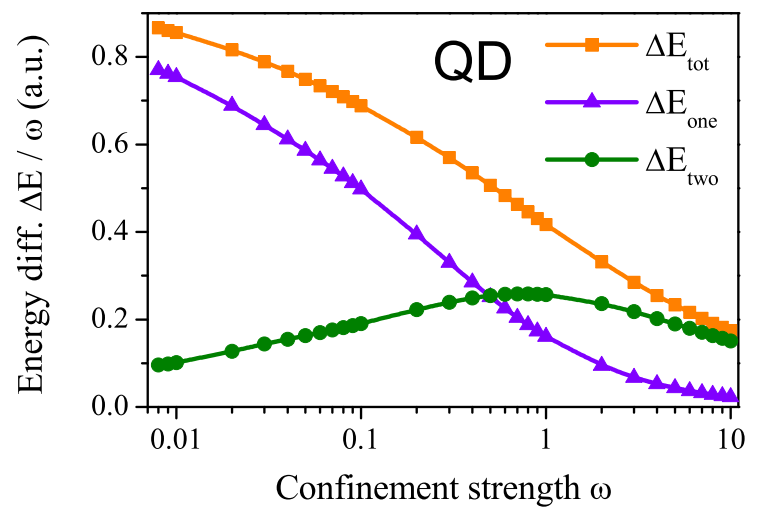

Figure 1. (Colour online) Energy differences (in hartree) between the lowest $P$ singlettriplet pair of states of He-like systems (upper figure) and of quantum dots (lower figure) for different nuclear charges $Z_{\mathrm{n}}$ and different strengths of confinement $\omega$. The difference in the total, one-electron and two-electron energies is denoted by $\Delta E_{\text {tot }}$, $\Delta E_{\text {one }}$ and $\Delta E_{\text {two }}$, respectively.

singlet and triplet states, i.e., $\Delta E_{\text {tot }} \equiv{ }^{1} E_{\text {tot }}-{ }^{3} E_{\text {tot }}, \Delta E_{\text {one }} \equiv{ }^{1} E_{\text {one }}-{ }^{3} E_{\text {one }}$, and $\Delta E_{\text {two }}$ $\equiv{ }^{1} E_{\text {two }}-{ }^{3} E_{\text {two }}$, respectively, are plotted as a function of $Z_{n}$ and $\omega$. Clearly, $\Delta E_{\text {tot }}$ $=\Delta E_{\text {one }}+\Delta E_{\text {two }}$. In all cases the energies of the scaled Hamiltonians, $\mathcal{H}_{Z} / Z_{\mathrm{n}}^{2}$ and $\mathcal{H}_{\omega} / \omega$, are plotted so that the results for different values of $Z_{\mathrm{n}}$ and $\omega$, respectively, can be compared on the same energy scale [see Eqs. (5) and (6)].

Figure 1 shows that for large $Z_{\mathrm{n}}$ and $\omega$ values, such as for example $Z_{\mathrm{n}}=20$ and $\omega=10$, respectively, the singlet-triplet energy gap $\Delta E_{\text {tot }}$ is dominated by the two-electron contribution $\Delta E_{\text {two }}$, which is apparently in accord with the traditional interpretation based on Slater's paper [9]. However, with decreasing $Z_{\mathrm{n}}$ and $\omega$ the twoelectron contribution becomes eventually smaller than the one-electron contribution which steadily increases. This is due to the so-called orbital relaxation $[10,11,32]$ : As implied by Eqs. (5) and (6) the electron-electron interaction becomes stronger relative to the one-electron component as $Z_{\mathrm{n}}$ and $\omega$ decrease, respectively. Therefore, when the 

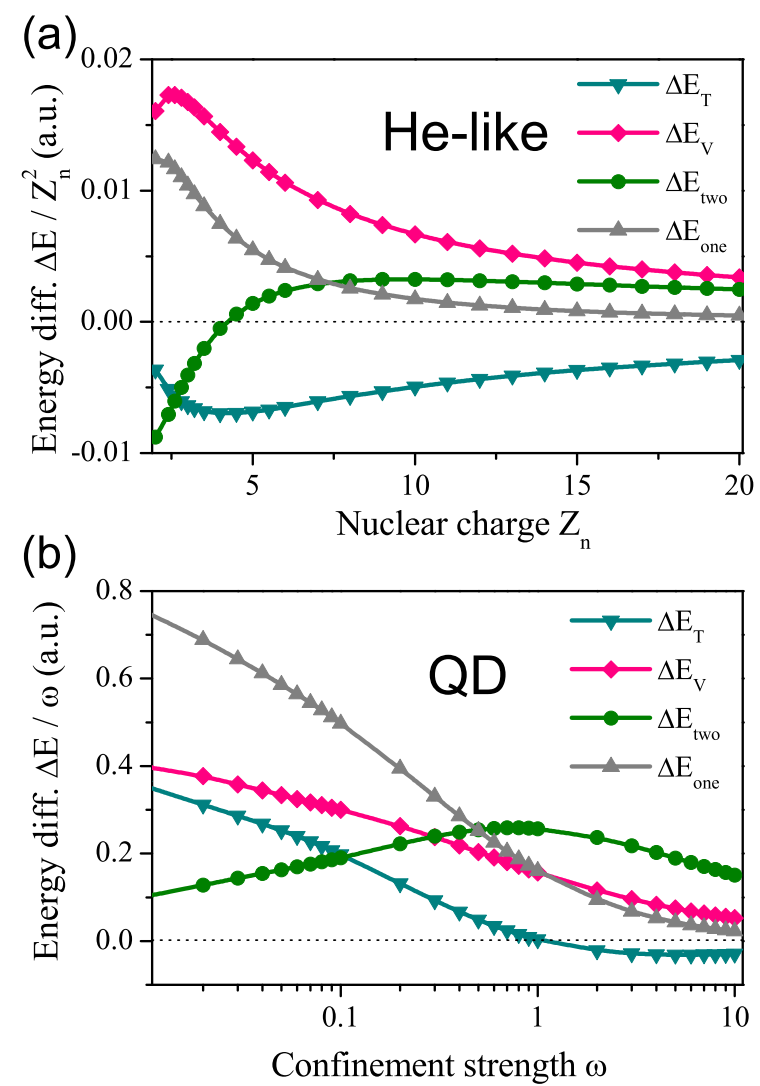

Figure 2. (Colour online) Kinetic energy and one-electron potential energy contributions to the singlet-triplet energy gap for the lowest $P$ singlet-triplet pair of states of He-like systems (upper figure) and of quantum dots (lower figure) for different nuclear charges $Z_{\mathrm{n}}$ and strengths of confinement $\omega$. The difference in the kinetic and one-electron potential energies is labeled by $\Delta E_{\mathrm{T}}$ and $\Delta E_{\mathrm{V}}$, respectively, while the symbols $\Delta E_{\text {one }}$ and $\Delta E_{\text {two }}$ refer, respectively, to the difference in the oneand two-electron energies taken from Fig. 1.

Hartree-Fock orbitals are independently optimized for the singlet and triplet states the resultant orbitals for different spin states become more and more distinct for smaller $Z_{\mathrm{n}}$ and $\omega$ resulting in a larger difference of the one-electron energy as is evidenced by the increase of $\Delta E_{\text {one }}$ in Fig. 1.

For later discussion it is useful to define regimes of $Z_{\mathrm{n}}$ and $\omega$ where the traditional interpretation is valid and where it breaks down. As already observed in Fig. 1 the traditional interpretation seems to be working in the regime of large $Z_{\mathrm{n}}$ and $\omega$ where the difference in the electron repulsion energy $\Delta E_{\text {two }}$ dominates the singlet-triplet energy gap. As suggested by Katriel [44], it is instructive to separate the one-electron energy component $\Delta E_{\text {one }}$ into its kinetic and one-electron potential components. The result is displayed in Fig. 2 where the symbols $\Delta E_{\mathrm{T}}$ and $\Delta E_{\mathrm{V}}$ represent the difference in the kinetic and one-electron potential energies, respectively, between the singlet and triplet 
(a)

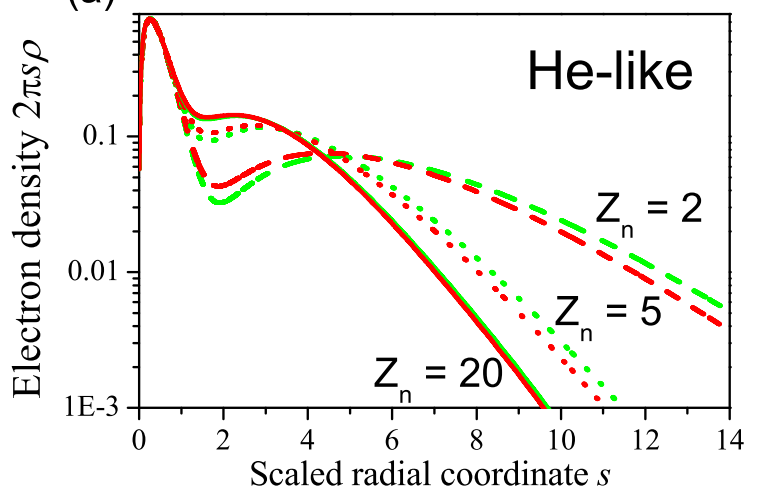

(b)

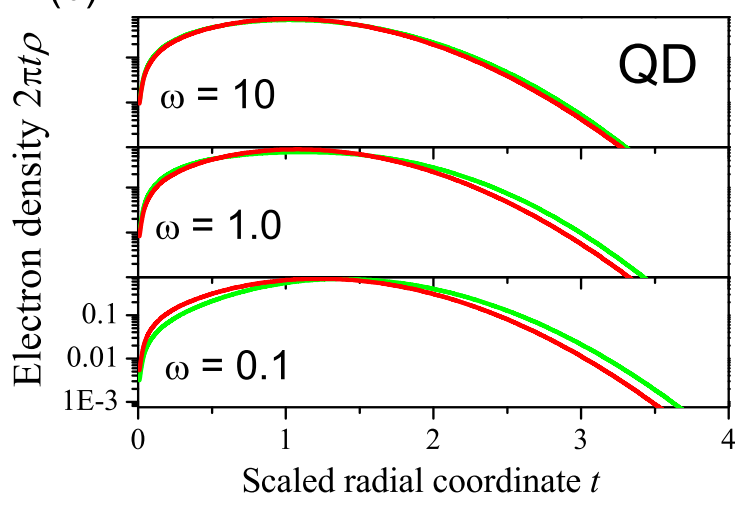

Figure 3. (Colour online) Radial electron density distributions of the lowest $P$ singlet-triplet pair of states of He-like systems (upper figure) and of quantum dots (lower figure) for $Z_{\mathrm{n}}=2,5$ and 20 and $\omega=0.1,1.0$ and 10 . The singlet and triplet densities are colored green and red (light-grey and dark-grey in black-and-white version), respectively. The $Z_{\mathrm{n}}$-scaled $s$ coordinate is used for He-like systems while the $\omega$-scaled $t$ coordinate is used for quantum dots (see the text for details).

states. In the same figure the one- and two-electron energy components $\Delta E_{\text {one }}$ and $\Delta E_{\text {two }}$ are plotted for comparison. The results for He-like systems shows that the nuclear attraction energy component, $\Delta E_{\mathrm{V}}$, is larger than the difference in the electron repulsion energy $\Delta E_{\text {two }}$ even at large $Z_{\mathrm{n}}$ values such as $Z_{\mathrm{n}}=20$. Yet, this positive contribution is almost canceled by the negative contribution of the kinetic energy component $\Delta E_{\mathrm{T}}$ and results in a very small one-electron contribution at large $Z_{\mathrm{n}}$. Therefore, it can be concluded that the traditional interpretation that ascribes the singlet-triplet energy gap solely to the two-electron contribution fails even in the large $Z_{\mathrm{n}}$ regime. On the other hand, the result for quantum dots in the large $\omega$ regime shows a one-electron potential contribution $\Delta E_{\mathrm{V}}$ to be much smaller than the two-electron energy component $\Delta E_{\text {two }}$, in contrast to the He-like systems. Therefore, in case of the quantum dots the traditional interpretation 'works' in the large $\omega$ regime. 
An examination of the one-electron density distribution of the relevant states helps to simplify the argument: In Figs. 3(a) and (b) the radial electron density distribution of the $(1 s)(2 p)$ and $\left(0 \sigma_{g}\right)\left(1 \pi_{u}\right)$ singlet-triplet pairs of states obtained from the FCI wave functions is plotted, respectively, for $Z_{\mathrm{n}}=2,5$ and 20 and $\omega=0.1,1.0$ and 10. In these figures the $Z_{\mathrm{n}^{-}}$and $\omega$-scaled radial coordinates $s$ and $t$ are used, respectively, and the electron densities are plotted in a logarithmic scale so that small differences between the states are clearly visible. For small and medium values of $Z_{\mathrm{n}}$ and $\omega$, i.e., $Z_{\mathrm{n}}=$ 2 and 5 , and $\omega=0.1$ and 1.0, the singlet and triplet densities colored green and red (light-grey and dark-grey in black-and-white version), respectively, are distinct. This is consistent with a significant contribution of the one-electron energy component $\Delta E_{\text {one }}$ in the regime of small and medium $Z_{\mathrm{n}}$ and $\omega$ as displayed in Fig. 1. For the regime of large $Z_{\mathrm{n}}$ and $\omega\left(Z_{\mathrm{n}}=20\right.$ and $\left.\omega=10\right)$, however, the electron density distribution of the singlet and triplet states almost coincide with each other. This is also consistent with the very small one-electron contribution $\Delta E_{\text {one }}$ and the dominating two-electron contribution $\Delta E_{\text {two }}$. Therefore, in He-like systems as well as in quantum dots the difference in the electron density distribution between the singlet and triplet pair of states agrees with the difference in the one-electron energy and thus the two-electron energy: In the regime of large $Z_{\mathrm{n}}$ and $\omega$ the small difference in the electron density distribution gives a small difference in the one-electron energy (the two-electron energy dominates the singlet-triplet energy gap) while for the medium and small regimes a large difference in the electron density distributions gives rise to a large difference in the one-electron energy (a breakdown of the traditional interpretation). On the other hand, when the one-electron contribution $\Delta E_{\text {one }}$ is partitioned into its kinetic energy and one-electron potential energy components $\Delta E_{\mathrm{T}}$ and $\Delta E_{\mathrm{V}}$ as displayed in Fig. 2, it splits into a positive $\Delta E_{\mathrm{V}}$ and a negative $\Delta E_{\mathrm{T}}$ even in the regime of large $Z_{\mathrm{n}}$ and $\omega$ regime that result in a very small one-electron contribution. Further, in this large $Z_{\mathrm{n}}$ and $\omega$ regime the one-electron potential contribution $\Delta E_{\mathrm{V}}$ is larger than the twoelectron contribution $\Delta E_{\text {two }}$ for He-like systems but is much smaller for quantum dots as has been shown in Fig. 2. Therefore, based on an analysis of the energy components it is difficult to decide whether the traditional interpretation is valid or not in the regime of large $Z_{\mathrm{n}}$ and $\omega$. In the following discussion we adopt the convention introduced in our previous studies $[32,31]$ to refer to the validity of the traditional interpretation: For the regime of large $Z_{\mathrm{n}}$ and $\omega$ regime the traditional interpretation works while it breaks down in the regime of medium and small $Z_{\mathrm{n}}$ and $\omega$. In Sec. 3.3 the difference in the magnitude of the one-electron potential contribution $\Delta E_{\mathrm{V}}$ relative to the two-electron contribution $\Delta E_{\text {two }}$ between He-like systems and quantum dots is rationalized.

Returning to the observations made in Fig. 1, a fundamental difference between Helike systems and quantum dots is most apparent from the behavior in the regime of small $Z_{\mathrm{n}}$ and $\omega$ : In the small $\omega$ regime of quantum dots the two-electron contribution $\Delta E_{\text {two }}$ monotonically decreases with decreasing $\omega$ and approaches zero in the limit $\omega \rightarrow 0$. In the case of He-like systems the two-electron contribution does indeed vanish around $Z_{\mathrm{n}}=4$ and becomes significantly negative at $Z_{\mathrm{n}}=2$. Consequently, the two-electron 


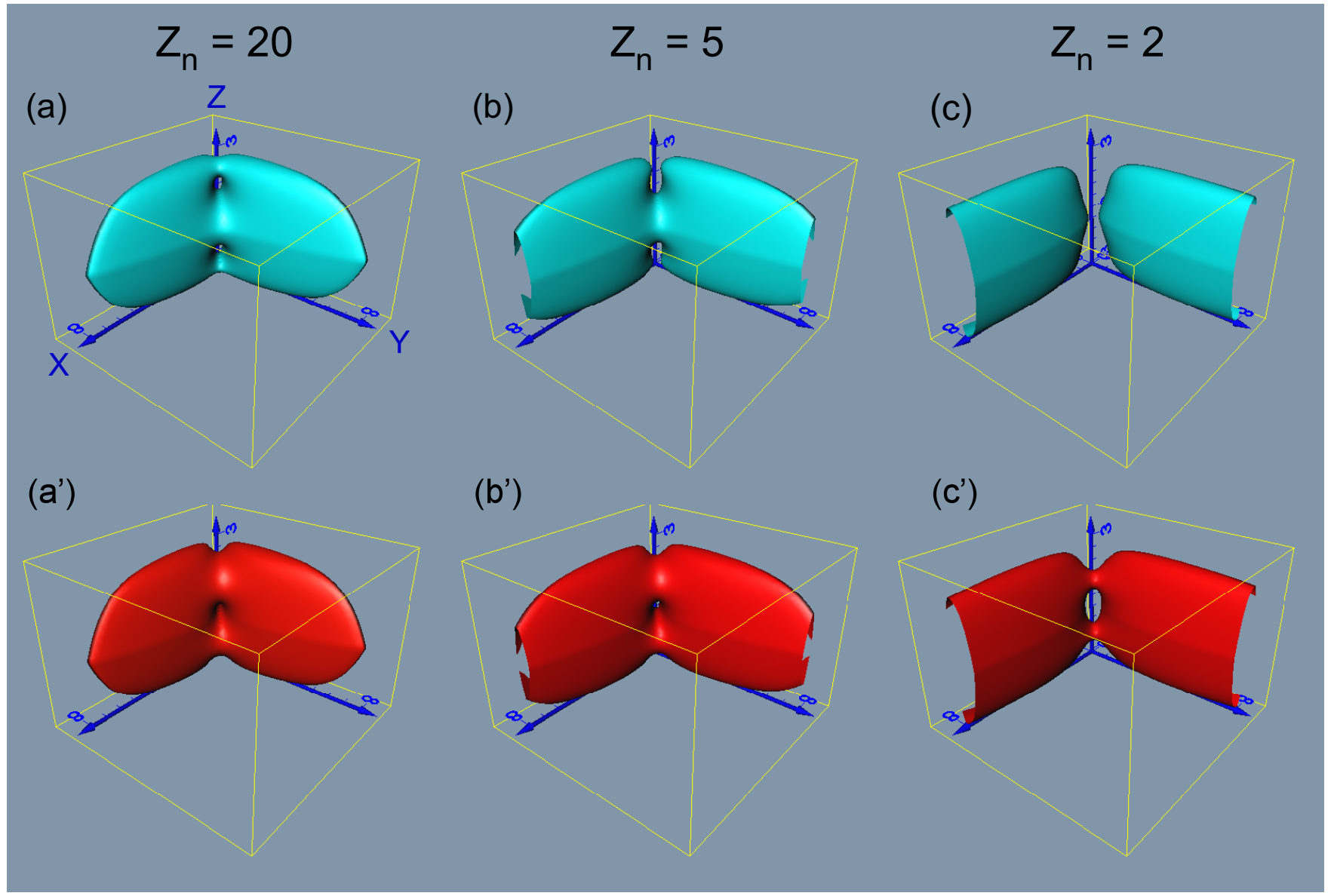

Figure 4. (Colour online) Isosurfaces of the probability density for the internal wave functions for the $(1 s)(2 p)$ singlet-triplet pair of the helium atom and helium-like ions. Figures (a), (b) and (c) represent the singlet wave functions with $Z_{\mathrm{n}}=20,5$ and 2 , respectively, while figures (a'), (b') and (c') represent the corresponding triplet wave functions. The square-norm of the displayed surface is 0.002. In all six figures the right-handed Cartesian coordinates $X, Y$ and $Z$, indicated in figure (a) correspond, respectively, to $Z_{\mathrm{n}}$-adjusted internal coordinates $s_{1}, s_{2}$ and $\phi_{-}$. The origin of the coordinate system is $(X, Y, Z)=(0,0,0)$.

energy of the triplet state exceeds that of the corresponding singlet, as first reported by Davidson [10, 11].

\subsection{Internal wave functions}

In order to understand the fundamental difference between He-like systems and quantum dots observed in the previous section, namely the fact that for the latter systems the electron repulsion energy of the triplet state stays smaller than that of the corresponding singlet state, yet can be larger in the He-like systems for small values of $Z_{\mathrm{n}}$, we explored the internal wave functions of both systems. The results are displayed in Figs. 4 and 5 where the internal wave functions of the $(1 s)(2 p)$ singlet-triplet pair of states for Helike systems and those of the $\left(0 \sigma_{g}\right)\left(1 \pi_{u}\right)$ pair of states for quantum dots are plotted, 


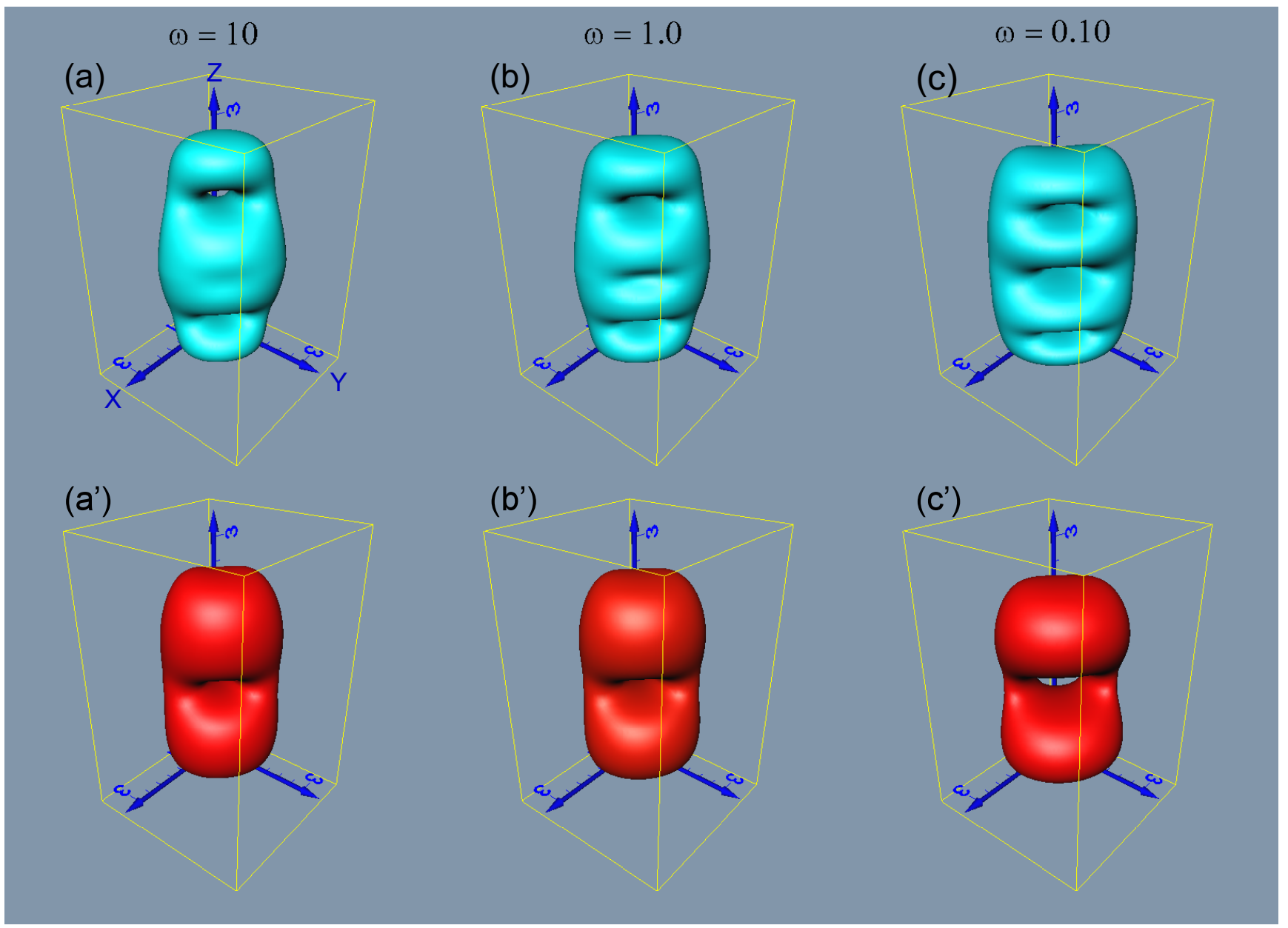

Figure 5. (Colour online) Isosurfaces of the probability density for the internal wave functions for the $\left(0 \sigma_{g}\right)\left(1 \pi_{u}\right)$ singlet-triplet pair of a quantum dot. Figures (a), (b) and (c) represent the singlet wave functions with $\omega=10,1$ and 0.1 , respectively, while figures (a'), (b') and (c') represent the corresponding triplet wave functions. The square-norm of the displayed surface is 0.01. In all six figures the right-handed Cartesian coordinates $X, Y$ and $Z$, indicated in figure (a) correspond, respectively, to the $\omega$-adjusted internal coordinates $t_{1}, t_{2}$ and $\phi_{-}$. The origin of the coordinate system is $(X, Y, Z)=(0,0,0)$.

respectively, for $Z_{\mathrm{n}}=20,5$, and 2 and $\omega=10,1$, and 0.1 . The standard right-handed $X$, $Y$, and $Z$ coordinates that are used in these plots correspond to the $Z_{\mathrm{n}^{-}}$and $\omega$-adjusted internal coordinates $t_{1}, t_{2}$ and $\phi_{-}$for He-like systems and $s_{1}, s_{2}$ and $\phi_{-}$for quantum dots, respectively. It is remarkable that when neglecting electron-electron interaction the internal wave functions in these scaled coordinate systems for different $Z_{\mathrm{n}}$ and $\omega$ appear to be identical with one another. Thus, it is important to examine the differences in the probability density distributions among internal wave functions with different $Z_{\mathrm{n}}$ and $\omega$ which reveal the role of the electron repulsion potential in the nodal structure of the internal wave functions. The domains for these internal coordinates in these figures are $0 \leq s_{i} \leq 8(i=1,2)$ and $-\pi \leq \phi_{-} \leq \pi$ for Fig. 4 and $0 \leq t_{i} \leq 3(i=1,2)$ and $-\pi \leq \phi_{-} \leq \pi$ for Fig. 5 (we note that in these and the following figures the scale of the 
$\phi_{-}$-axis is labeled numerically in radians rather than fractions of $\pi$, e.g., $\pi / 2$, etc.). The density of the displayed surfaces has been chosen as 0.002 and 0.01 for Figs. 4 and 5, respectively.

As shown in Fig. 1 the effect of the electron-electron interaction is very small for large values of $Z_{\mathrm{n}}$ and $\omega$ such as $Z_{\mathrm{n}}=20$ and $\omega=10$, respectively, since the difference in the one-electron energy $\Delta E_{\text {one }}$ between the singlet-triplet pair of states is very small, indicating that the electron repulsion is not strong enough to induce a meaningful orbital relaxation. Therefore, the internal wave functions (a) and (a') in Fig. 4 for $Z_{\mathrm{n}}=20$ and those in Fig. 5 for $\omega=10$ are very much alike the zeroth order internal wave functions constructed from unoptimized $(1 s)$ and $(2 p)$ atomic orbitals without taking electron repulsion into account. The small density areas observed as vacancies on the surfaces at around $Z=0$ for the triplet and around $Z= \pm \pi / 2$ for the singlet state both for the He-like systems and quantum dots are thus inherent to the $(1 s)(2 p)$ and $\left(0 \sigma_{g}\right)\left(1 \pi_{u}\right)$ configurations. These small density areas represent the so-called Fermi holes and conjugate Fermi holes, and are discussed in detail in the next section.

The following observations that can be made on the basis of the internal wave functions displayed in Figs. 4 and 5 are pointed out: First, the internal wave functions of the He-like systems have a 'wing' like shape that extents along the $X$ and $Y$ axes, while those of quantum dots have a round shape with a large probability density in the direction of simultaneously increasing $X$ and $Y$ axes, i.e. in the direction parallel to the plane defined by $X=Y$. This difference in the shape of the internal wave functions of these two systems can be understood by considering the spatial extent of the relevant one-electron orbitals: In the case of the He-like atomic systems there is a large difference in the size of the $(1 s)$ and $(2 p)$ orbitals, since they belong to different principal shells of $n=1$ and $n=2$. On the other hand, in the case of quantum dots, the $\left(0 \sigma_{g}\right)$ and $\left(1 \pi_{u}\right)$ orbitals also reside in different shells of $v_{p}=0$ and 1 where the polyad quantum number $v_{p}$ accounts for the shell structure of the two-dimensional harmonic oscillator $[40,45,32,46]$, yet their spatial extent is of the same order of magnitude since the classical turning points of the harmonic oscillator along the $x$ axis, for example, are $\left|x_{\mathrm{c}}\right|$ $=1$ and $\sqrt{3}$ for $v=0$ and 1 , respectively.

In the zeroth-order approximation the orbital part of the two-electron wave functions can be expressed as a single determinant

$$
\Psi^{ \pm}=\frac{1}{\sqrt{2}}\left[\psi_{a}\left(\vec{r}_{1}\right) \psi_{b}\left(\vec{r}_{2}\right) \pm \psi_{b}\left(\vec{r}_{1}\right) \psi_{a}\left(\vec{r}_{2}\right)\right]
$$

where $\Psi^{+}$and $\Psi^{-}$correspond to, respectively, the singlet and triplet state (strictly speaking, the orbitals $\psi_{a}$ and $\psi_{b}$ are distinct for the singlet and triplet states when independently optimized [10, 11], but the argument here is not affected by this difference). As implied by Eq. (9) there are two terms contributing to the probability density distribution, namely, $\psi_{a}\left(\vec{r}_{1}\right) \psi_{b}\left(\vec{r}_{2}\right)$ and $\psi_{b}\left(\vec{r}_{1}\right) \psi_{a}\left(\vec{r}_{2}\right)$. The orbital subscripts $a$ and $b$ correspond to $1 s$ and $2 p$ for He-like systems and to $0 \sigma_{g}$ and $1 \pi_{u}$ for quantum dots. Then, the probability densities $\left|\psi_{1 s}\left(\vec{r}_{1}\right) \psi_{2 p}\left(\vec{r}_{2}\right)\right|^{2}$ and $\left|\psi_{2 p}\left(\vec{r}_{1}\right) \psi_{1 s}\left(\vec{r}_{2}\right)\right|^{2}$ for He-like systems represent, respectively, the right and left 'wings' of the internal wave functions 
as displayed in Fig. 4. The right wing has a broad distribution along the $Y$ axis, i.e., the radial coordinate of electron 2 , but it has a narrow distribution along the $X$ axis, i.e., the radial coordinate of electron 1 . The shape of this right wing thus reflects the fact that electrons 1 and 2 occupy, respectively, narrow $(1 s)$ and broad $(2 p)$ orbitals. The same argument can be applied to the relationship between $\left|\psi_{2 p}\left(\vec{r}_{1}\right) \psi_{1 s}\left(\vec{r}_{2}\right)\right|^{2}$ and the left wing.

In the case of quantum dots both probability densities $\left|\psi_{0 \sigma_{g}}\left(\vec{r}_{1}\right) \psi_{1 \pi_{u}}\left(\vec{r}_{2}\right)\right|^{2}$ and $\left|\psi_{1 \pi_{u}}\left(\vec{r}_{1}\right) \psi_{0 \sigma_{g}}\left(\vec{r}_{2}\right)\right|^{2}$ distribute along the $X$ and $Y$ axes to a similar extent, since the spatial distribution of the two orbitals, $\psi_{0 \sigma_{g}}$ and $\psi_{1 \pi_{u}}$, is of the same order, in contrast to the $(1 s)$ and $(2 p)$ orbitals of Coulomb systems. Consequently, the internal wave functions of quantum dots have a cylindrical shape as seen in Fig. 5. These differences in the spatial distribution of the internal wave functions for He-like systems and quantum dots explain the above noted difference of the two-electron contribution $\Delta E_{\text {two }}$ as will be pointed out in greater detail in the next section.

Second, the internal wave functions of both singlet and triplet states for He-like systems become broader as the nuclear charge $Z_{\mathrm{n}}$ decreases. As seen in Figs. 4(b) and $4\left(b^{\prime}\right)$ the edge of the internal wave functions for $Z_{\mathrm{n}}=5$ slightly touches the chosen domain for the $X$ and $Y$ axes, i.e., the planes defined by $X=8$ and $Y=8$, respectively, revealing an edge of a thin layer plot representing the resulting cross sections (note that the interior of the displayed surfaces of the internal wave functions is not vacant as the plots (b) and (b'), as well as (c) and (c'), of Fig. 4 would seem to suggest but involves a non-zero probability density that is larger than that for the displayed surfaces. Since the internal wave functions are represented by an isosurface of a selected value of probability density, the graphical software does not plot points whose density is different from the selected value). On the other hand, such cutoffs are not seen for the corresponding wave functions (a) and (a') for $Z_{\mathrm{n}}=20$, while they are enhanced for the wave function (c) for $Z_{\mathrm{n}}=2$ relative to $Z_{\mathrm{n}}=5$, indicating that the size of the internal wave functions increases with decreasing value of $Z_{\mathrm{n}}$. Thus, as $Z_{\mathrm{n}}$ decreases the probability density migrates from the region where both $s_{1}$ and $s_{2}$ are small towards the region where independently $s_{1}$ or $s_{2}$ are large. It is also noted that the singlet wave function has a larger cross section than does the corresponding triplet one as may be seen most clearly from Figs. 4(b) and 4(b'). This indicates that the internal wave function of the triplet state is more compact than that of the singlet, confirming the results of earlier studies $[32,31]$. In case of quantum dots the size of the internal wave functions becomes larger as $\omega$ decreases similarly as for He-like systems, but the difference for different $\omega$ values is not as large as in the He-like case for different $Z_{\mathrm{n}}$.

Third, for both He-like systems and quantum dots in the regime of large $Z_{\mathrm{n}}$ and $\omega$, respectively, the singlet and the corresponding triplet wave functions reveal distinct nodal structures. For example, as seen in Figs. 4(a) and 4(a') as well as in Figs. 5(a) and 5(a') the singlet wave functions have nodes at around $Z= \pm \pi / 2$ while the corresponding triplet wave functions have a node at around $Z=0$. Since the effect of the electron repulsion potential on the internal wave functions should be very small 
in the regime of large $Z_{\mathrm{n}}$ and $\omega$, the difference in the nodal patterns between the singlet and triplet internal wave functions must be ascribed solely to the antisymmetrization of the wave functions as implied by Eq. (9). Further, the singlet wave function of He-like system with $Z_{\mathrm{n}}=20$ and that of quantum dots with $\omega=10$ has a large probability density at around $Z=0$ where the corresponding triplet wave function has a node. As $Z_{\mathrm{n}}$ and $\omega$ decrease, however, the singlet probability density in this region decreases as well, leading to a clearly visible node for smaller $Z_{\mathrm{n}}$ and $\omega$ [Fig. 4(c) and Fig. 5(c)]. This is the crucial effect due to the electron repulsion potential as examined in detail in the next section in terms of the genuine and conjugate Fermi hole concept.

\subsection{Structure of the genuine and conjugate Fermi holes}

In order to rationalize the observations made in the previous section, the difference between the probability densities of the singlet and triplet internal wave functions has been calculated and displayed in Figs. 6 and 7 for He-like systems and quantum dots, respectively. In these figures the scaled electron-electron interaction potentials, $\frac{1}{Z_{\mathrm{n}}\left|\vec{s}_{1}-\vec{s}_{2}\right|}$ and $\frac{1}{\sqrt{\omega}\left|\vec{t}_{1}-\vec{t}_{2}\right|}$, have been also displayed as an isosurface with the energy value chosen as 0.5 and 3.0 a.u. for Figs. 6 and 7, respectively. It is seen that the electron-electron interaction potential manifests itself as three striking poles peaked at $Z=0, \pm \pi$, i.e., $\phi_{-}$ $=0, \pm \pi[31]$. All these three internal angles correspond to the same spatial configuration in which the two electrons are aligned on the same side of the nucleus. Therefore, when their radial distance from the nucleus is the same, i.e., when $X=Y$, their electron repulsion potential diverges, resulting in a very large energy in the vicinity of these three poles. Since the electron repulsion potential in the scaled internal spaces is inversely proportional to $Z_{\mathrm{n}}$ and $\sqrt{\omega}$, it becomes larger as $Z_{\mathrm{n}}$ or $\omega$ decreases as seen in Figs. 6 and 7 , respectively.

The plots (a) - (c) in Figs. 6 and 7 representing the difference between the probability densities of the singlet and triplet internal wave functions are colored in blue and red (light-grey and dark-grey in black and white version). These colors correspond, respectively, to the regions in which the singlet state has a larger probability density than the corresponding triplet, and vice versa. For the regime of large $Z_{\mathrm{n}}$ and $\omega$ [e.g., case (a) in Figs. 6 and 7] the blue regions are located around the regions in which the electron repulsion potential diverges, i.e., in regions defined by the equations $X=Y$ and $Z=0, \pm \pi$. Therefore, these blue regions represent the so-called Fermi holes. Since the triplet electrons are keeping away from the Fermi holes while the singlet electrons are not, the singlet probability density in the vicinity of Fermi holes should be larger than the triplet one resulting in the observed blue regions. On the other hand, the red regions represent the so-called conjugate Fermi holes [31] from which the singlet electrons are repelled while the triplet electrons are not.

The origin of the appearance of genuine and conjugate Fermi holes can thus be briefly described as follows: For the $(1 s)(2 p)$ singlet-triplet pair we can write the zero- 


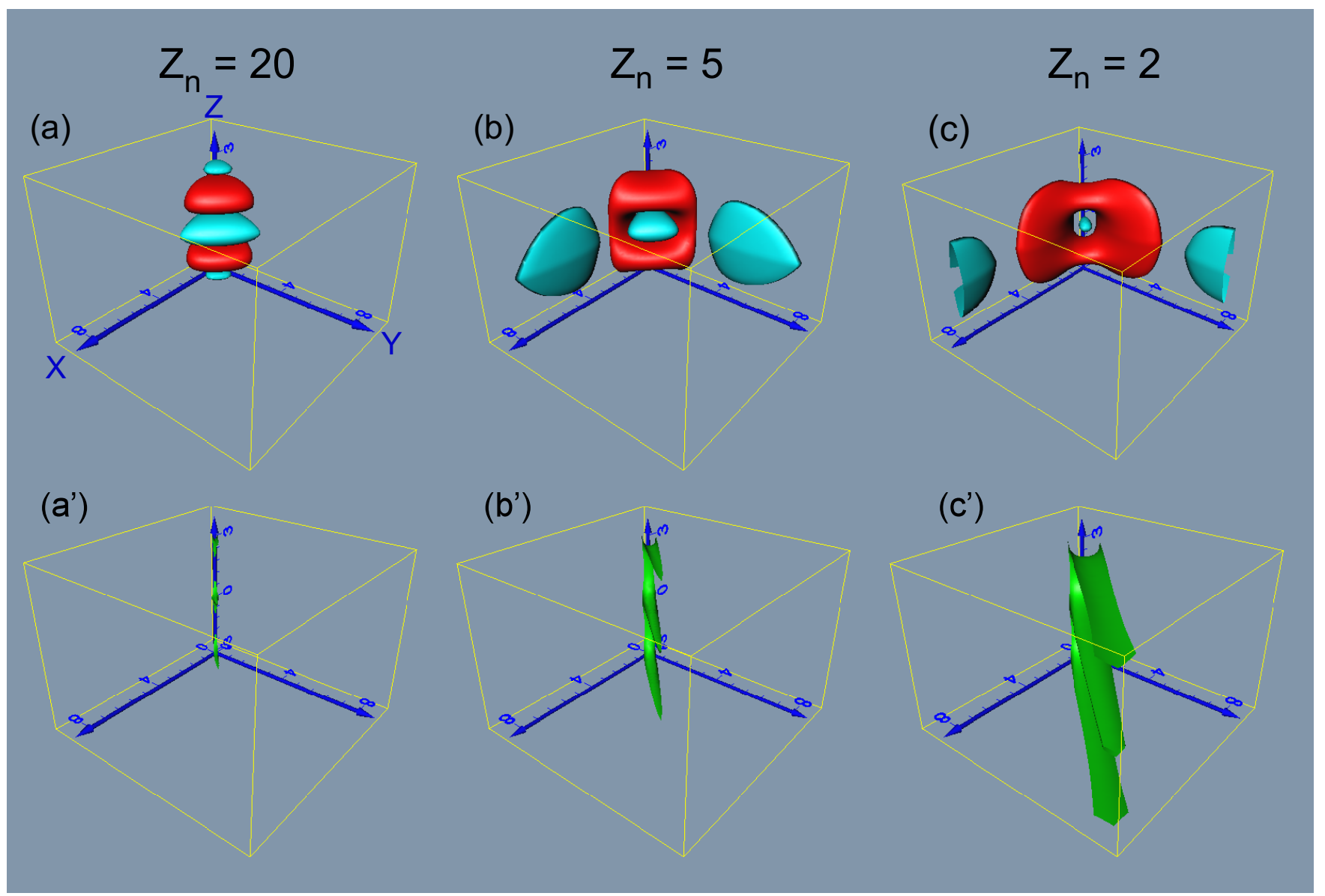

Figure 6. (Colour online) Difference between the probability density distributions of the $(1 s)(2 p)$ singlet state ${ }^{1} P$ and triplet state ${ }^{3} P$ of He-like systems in the internal space: Figures (a), (b) and (c) correspond to $Z_{\mathrm{n}}=20,5$ and 2, respectively. The square norm of the displayed surface is 0.001. The blue (light grey) and red (dark grey) surfaces correspond, respectively, to regions in which the probability density of the singlet wave function is larger than that of the triplet one and vice versa. Figures (a'), (b') and (c') indicate the electron repulsion potential for the corresponding cases whose displayed surfaces represent the area where the electron repulsion potential energy becomes larger than 0.5 a.u.. See the caption to Fig. 4 for further details.

order wave function of Eq. (9) in the following more explicit form

$$
\begin{aligned}
\Psi^{ \pm}= & \frac{1}{\sqrt{4 \pi}} \exp \left(i \phi_{+}\right)\left[\psi_{1 s}\left(r_{1}\right) \psi_{2 p}\left(r_{2}\right) \exp \left(-i \phi_{-}\right)\right. \\
& \left. \pm \psi_{2 p}\left(r_{1}\right) \psi_{1 s}\left(r_{2}\right) \exp \left(i \phi_{-}\right)\right],
\end{aligned}
$$

where only one component of the doubly-degenerate $(1 s)(2 p)$ states with the orbital angular momentum $L=1$ is accounted for. Since the other component with $L=-1$ yields the same internal wave function, the following discussion focuses only on the singlet-triplet pair of states with positive $L$. For the spatial configuration defined by $r_{1}=r_{2}$ and $\phi_{-}=0, \pm \pi$, that correspond to $\vec{r}_{1}=\vec{r}_{2}$, and thus to the case when the electron repulsion potential diverges, the triplet wave function $\Psi^{-}$vanishes, since the first and the second terms in the bracket of Eq. (10) cancel one another. In contrast, 


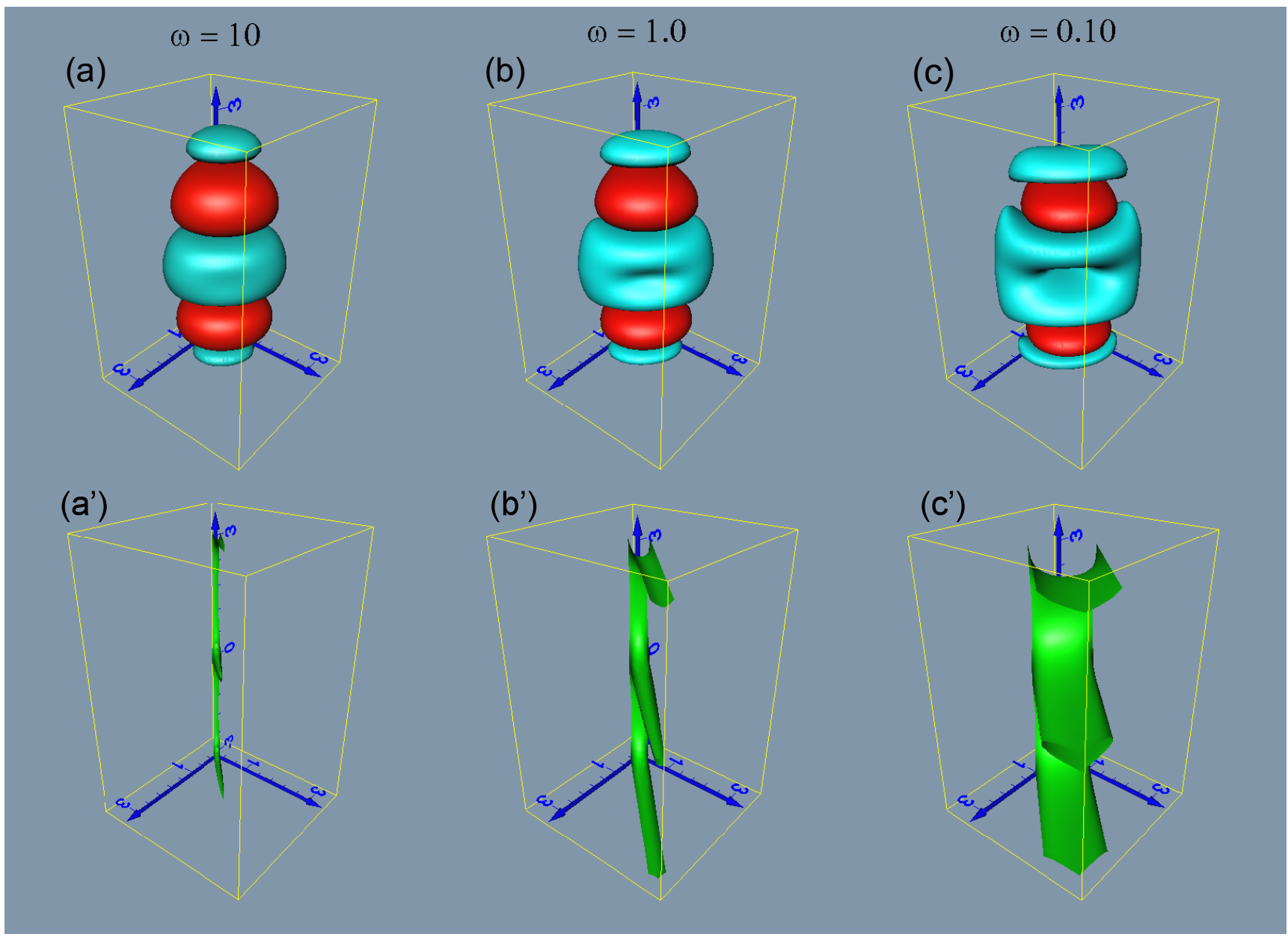

Figure 7. (Colour online) Difference between the probability density distributions of the $\left(0 \sigma_{g}\right)\left(1 \pi_{u}\right)$ singlet ${ }^{1} P$ and triplet ${ }^{3} P$ states of quantum dots in the internal space: Figures (a), (b) and (c) correspond to $\omega=10,1$ and 0.1 , respectively. The square norm of the displayed surface is 0.01 . The blue (light grey) and red (dark grey) surfaces correspond, respectively, to regions in which the probability density of the singlet wave function is larger than that of the triplet one and vice versa. Figures (a'), (b') and (c') represent the electron repulsion potential for the corresponding cases whose displayed surfaces indicate the area where the electron repulsion energy becomes larger than 0.3 a.u.. See the caption to Fig. 5 for further details.

the singlet wave function $\Psi^{+}$has a non-zero probability density in this case. Therefore, the singlet wave function always has larger probability densities than the corresponding triplet one near these spatial configurations. This is the origin of the appearance of the well-known Fermi holes as represented by the blue regions in Fig. 6(a).

Now, in turn, for the spatial configuration defined by $r_{1}=r_{2}$ and $\phi_{-}= \pm \pi / 2$, the singlet wave function $\Psi^{-}$vanishes since the phase factors that are associated with the first and the second terms in the bracket of Eq. (10) have different signs leading to a cancellation of these terms. In the case of the triplet wave function, however, this phase difference is compensated by the original minus sign in the bracket so that the first and the second terms are added together resulting in a nonzero probability density. This is 
the origin of the appearance of the conjugate Fermi hole represented by red regions in Fig. 6(a).

It must be emphasized that the appearance of the conjugate Fermi hole is not limited to the $(1 s)(2 p)$ configuration considered here but represents a general feature in the sense that genuine and conjugate Fermi holes always appear as a pair. In short, for the genuine and conjugate Fermi holes to appear there must exist, first of all, spatial regions in which the distinct one-electron orbitals appearing in Eq. (9) mutually overlap. Otherwise, there would be no energy difference between the singlet and triplet states. Then, the genuine hole will appear inside the overlapping region where $\vec{r}_{1}$ and $\vec{r}_{2}$ coincide, while the conjugate Fermi hole will appear inside this overlapping region where both of $\vec{r}_{1}$ and $\vec{r}_{2}$ are close to one of the node points $\vec{r}_{0}$ of one of the orbitals, i.e., a point satisfying $\psi_{a}\left(\vec{r}_{0}\right)=0$ or $\psi_{b}\left(\vec{r}_{0}\right)=0$, and where $\vec{r}_{1}$ and $\vec{r}_{2}$ do not coincide. Therefore, the nodal structure of the orbitals is essential for conjugate Fermi holes. Yet, since the orbitals $\psi_{a}$ and $\psi_{b}$ in Eq. (9) are distinct and orthonormal the number of nodes in these orbitals is different which guarantees the existence of at least one node in one of these orbitals.

We wish to emphasize that the distinction between the genuine and the conjugate Fermi holes is essential in the limit of large nuclear charge $Z_{\mathrm{n}}$ (or large $\omega$ for quantum dots) where the singlet and triplet wave functions can be represented in terms of the same set of one-electron orbitals (i.e., no orbital relaxation). Then, although these singlet and triplet wave functions yield the same one-electron density distributions, the triplet wave function has a "hole" in the region where the electron coordinates $\vec{r}_{1}$ and $\vec{r}_{2}$ coincide (genuine Fermi hole) while the singlet wave function is also characterized by a hole but in a different region determined by the nodal structure of the one-electron orbitals. We refer to the latter hole in the singlet wave function as the "conjugate Fermi hole" since its existence is closely related to the antisymmetrization of the electronic wave functions as for the genuine Fermi hole. A more detailed explanation of the origin of the appearance of conjugate Fermi holes with examples other than those involving $(1 s)(2 p)$ states was given in Ref. [31].

The plots in Fig. 6 that reveal the presence of the genuine and conjugate Fermi holes rationalize the origin of the first Hund rule in He-like systems as follows: In the plot (a) for $Z_{\mathrm{n}}=20$, representing the regime of large $Z_{\mathrm{n}}$ values, a large singlet probability density is found in the vicinity of regions characterized by Fermi holes where $X=Y$ and $Z=0$ while the triplet density is rather insignificant in these regions. Since the electron repulsion potential penetrates precisely into the Fermi holes as displayed in Fig. 6(a'), the singlet state should involve a larger electron repulsion than the triplet in accordance with the traditional interpretation based on the Slater's paper [9]. As $Z_{\mathrm{n}}$ decreases to $Z_{\mathrm{n}}=5$, however, the electron repulsion potential becomes stronger as displayed in Fig. 6(b'). Following this increase in the electron repulsion, a sizable portion of the singlet probability density starts to migrate out of the vicinity of the Fermi holes as displayed in Fig. 6(b) since the singlet probability density in these regions greatly increases the energy due to a strong electron repulsion. The fate of 
the migrated singlet probability density is then the key to the understanding of the origin of Hund's multiplicity rule. As displayed in Fig. 4 the internal wave functions of He-like systems extend along the $X$ and $Y$ axes due to the combination of the narrow $1 s$ and broad $2 p$ orbitals. Therefore, the singlet probability density that migrates out of the original regions is forced to move in the $X$ and $Y$ directions. On the other hand, since the conjugate Fermi holes surround the genuine Fermi holes as displayed in Figs. 6(a) and 6(b) which repel the singlet electrons, the singlet probability density has to migrate further away into the regions of either large $X$ or large $Y$.

As $Z_{\mathrm{n}}$ further decreases to $Z_{\mathrm{n}}=2$, the poles of the electron repulsion potential become so strong that most of the singlet probability density in the vicinity of the Fermi holes has to migrate into regions where the $X$ and $Y$ coordinates are independently large as seen in Fig. 6(c). Since these regions of large $X$ or large $Y$ are far away from the poles of the interelectronic repulsion potential as displayed in Fig. 6(c') the electron repulsion energy in the singlet state decreases to the extent that it becomes smaller than that of the corresponding triplet state. This leads to a negative value of the two-electron contribution to the singlet-triplet energy gap $\Delta E_{\text {two }}$ as observed in Fig. 1(a). Furthermore, the migrated singlet probability density is also sufficiently removed from the origin where the nucleus resides and the energy lowering due to the nuclear attraction potential becomes smaller and smaller for the singlet state relative to the corresponding triplet state as $Z_{\mathrm{n}}$ decreases. This again explains the fact why the one-electron contribution $\Delta E_{\text {one }}$ increases with decreasing $Z_{\mathrm{n}}$.

Historically, it has been often argued that the outer electron of the triplet state shrinks toward the nucleus yielding a more compact electron density distribution in the triplet state relative to the corresponding singlet state. The reason why the triplet state was deemed to be primarily responsible here may be associated with the fact that it has a different spin than the ground state. Since the ground state of He-like systems is singlet, it was considered to be well behaved while the triplet states were regarded as exceptional. However, it is actually the singlet state rather than the triplet one that is responsible for the complexity that is associated with a proper understanding of the origin of Hund's multiplicity rule: For small nuclear charges $Z_{\mathrm{n}}$ the electron density distribution in the singlet state extends over a much broader region than does the corresponding triplet distribution due to the existence of conjugate Fermi holes.

A similar argument can now be applied to the genuine and conjugate Fermi holes of quantum dots (cf. Fig. 7). The topological structure of these holes in the large $\omega$ regime of $\omega=10$ [Fig. $7(\mathrm{a})$ ] is similar to that of He-like systems in the large $Z_{\mathrm{n}}$ regime of $Z_{\mathrm{n}}=$ 20 [Fig. 6(a)]. However, their dissimilarity becomes apparent as $Z_{\mathrm{n}}$ and $\omega$ decrease: In the case of He-like systems the singlet probability density located in the regions defined by Fermi holes migrates towards the regions with large $X$ or $Y$ and appears as the left and right 'wing' of the probability density [Figs. 6(b) and (c)]. In the case of quantum dots, on the other hand, the singlet probability that is located in the vicinity of the Fermi holes does indeed migrate out of these regions as evidenced by an enlarged nodal region at $Z=0$ [Figs. 7(b) and (c)], but there appear no 'wings' for large $X$ and $Y$ that 
(a) $Z=20$

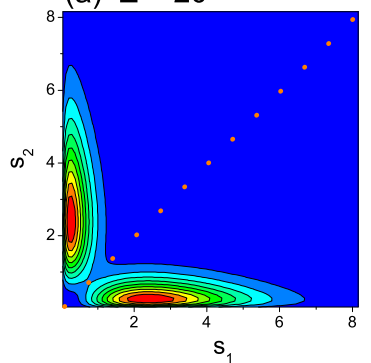

(a') $Z=2$

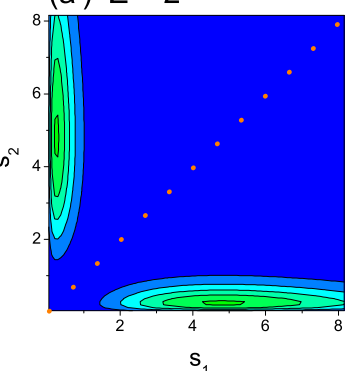

(b) $\omega=10$

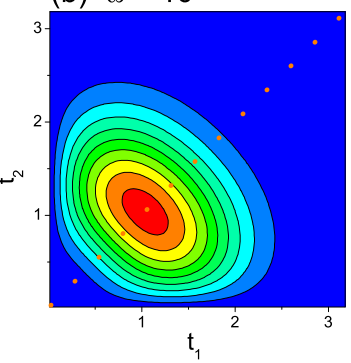

(b') $\omega=0.1$

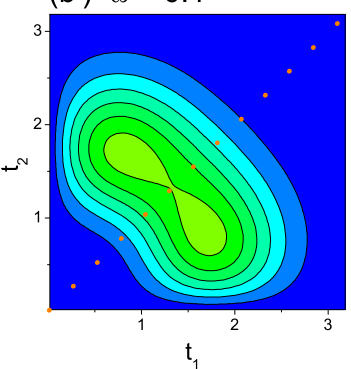

Figure 8. (Colour online) Projection of the internal wave functions onto the $X Y$ plane for the $(1 s)(2 p)$ singlet state of He-like systems and for the $\left(\sigma_{g}\right)\left(\pi_{u}\right)$ singlet state of quantum dots: Figures (a) and (a') represent the results for He-like systems with $Z_{n}$ $=20$ and 2, respectively, while figures (b) and (b') represent those for quantum dots with $\omega=10$ and 0.1 , respectively. The dotted diagonal line in each plot represents the line along which the electron repulsion potential diverges. See captions to Figs. 4 and 5 for the definitions of the coordinates $\left(s_{1}, s_{2}\right)$ and $\left(t_{1}, t_{2}\right)$, respectively.

characterize He-like atomic systems.

To identify the location of the singlet probability density that migrated out of the regions defined by Fermi holes in the case of quantum dots the internal wave functions of the singlet state displayed in Fig. 5 are integrated along the angle $Z$ coordinate and projected onto the $X Y$ plane. The results for $\omega=10$ and 0.1 are plotted in Fig 8. In the same figure the projection of the corresponding internal wave functions for He-like systems with $Z_{\mathrm{n}}=20$ and 2 are also shown for the sake of a comparison. The orange dotted diagonal line (light grey in black and white version) represents the line along which the electron repulsion potential diverges. For $Z_{\mathrm{n}}=20$ and $\omega=10$ the electron repulsion potential only slightly affects the probability density distributions since the electron repulsion potential in these cases is very small as seen in Figs. 6(a') and 7(a'). As $Z_{n}$ and $\omega$ decrease the electron repulsion potential becomes stronger and forces the singlet probability density in the vicinity of the Fermi holes to migrate out of these regions. In the case of He-like systems this singlet probability density migrates along the $s_{1}$ and $s_{2}$ axes [Fig. 8(a')] as explained earlier. On the other hand, in the case of quantum dots the singlet probability density located originally along the line $t_{1}=t_{2}$ migrates out of this region towards both sides of this line, as evidenced by the splitting of 
the highest density area along this diagonal line [Fig. 8(b')]. Unlike in He-like systems the migrated singlet probability density in quantum dots is not limited to regions of either large $t_{1}$ or $t_{2}$ but is spread out in a wide area where both $t_{1}$ and $t_{2}$ are large due to the similarity of the spatial extent of the $0 \sigma_{g}$ and $1 \pi_{u}$ orbitals. Therefore, in the case of quantum dots the singlet probability density that is located in the vicinity of Fermi holes does indeed avoid the line $t_{1}=t_{2}$ where the electron repulsion potential diverges yet still remains located in the surrounding region and thus yields a larger expectation value of the electron repulsion potential than the corresponding triplet state.

Finally, it is worthwhile to comment on the origin of the difference between He-like systems and quantum dots in the regime of large $Z_{\mathrm{n}}$ and $\omega$ values from the viewpoint of the magnitude of the one-electron potential energy contribution $\Delta E_{\mathrm{V}}$ relative to the two-electron contribution $\Delta E_{\text {two }}$ as represented in Fig. 2. As shown in Sec. 3.1, $\Delta E_{\mathrm{V}}$ is larger than $\Delta E_{\text {two }}$ for He-like systems but is much smaller for quantum dots. This observation can also be rationalized on the basis of the discussion made so far in this section: In the large $Z_{\mathrm{n}}$ and $\omega$ regime the electron repulsion potential at its poles is very small [cf. Figs. 6(a') and 7(a')], yet it can push a small portion of the singlet probability density out of the Fermi holes. In the case of He-like systems the singlet probability that migrated out of the Fermi holes is far away both from the poles of the electron repulsion potential and nucleus, yielding a smaller electron repulsion energy and a smaller absolute value of the nuclear attraction energy than is the case when the singlet probability density remains unchanged. However, since the absolute magnitude of the nuclear attraction potential is much larger than that of the electron repulsion potential thanks to the large $Z_{\mathrm{n}}$ factor, the difference in the nuclear attraction energy is larger than the electron repulsion energy, giving a larger $\Delta E_{\mathrm{V}}$ than $\Delta E_{\text {two }}$. On the other hand, in the case of quantum dots the singlet probability density that migrated out of the Fermi holes is still in the neighborhood of the original region so that the difference in the one-electron potential energy $\Delta E_{\mathrm{V}}$ cannot be large enough as to be compared with the two-electron energy difference $\Delta E_{\text {two }}$.

\section{Summary}

The focus of the present study is the origin of Hund's multiplicity rule in singly-excited states of 2D He-like systems and 2D quantum dots. The full configuration interaction (FCI) method has been employed to generate the energy spectrum and to partition the total energy into its one- and two-electron components. The internal part of the corresponding wave functions was generated from these FCI wave functions for the $(1 s)(2 p)$ and $\left(0 \sigma_{g}\right)\left(1 \pi_{u}\right)$ singlet-triplet pair for states of He-like systems and quantum dots, respectively, and has been visualised in the internal space $\left(r_{1}, r_{2}, \phi_{-}\right)$.

For the regime of large confinement, i.e., for large values of either the nuclear charge $Z_{\mathrm{n}}$ in He-like systems or of the harmonic frequency $\omega$ in quantum dots the partitioning of the singlet-triplet energy difference $\Delta E_{\text {tot }}$ into the one- and two-electron components, $\Delta E_{\text {one }}$ and $\Delta E_{\text {two }}$, shows a similar trend for both the He-like systems and the quantum 
dots. However, for the regime of small $Z_{\mathrm{n}}$ and $\omega$ their behavior is sufficiently distinct: the sign of $\Delta E_{\text {two }}$ stays positive for all $\omega$ values in quantum dots while it changes from positive to negative values for small $Z_{\mathrm{n}}$ in He-like systems indicating that the electron repulsion becomes smaller in the singlet state than in its triplet state.

In order to rationalize these distinct trends of $\Delta E_{\text {two }}$ for small $Z_{\mathrm{n}}$ and $\omega$ we rely on the structure of the conjugate Fermi holes as well as of the genuine Fermi holes in the internal space. Their structure is made apparent by considering the difference between the probability densities of the singlet and triplet internal wave functions. The genuine Fermi holes appear in regions defined by $r_{1}=r_{2}$ and $\phi_{-}=0, \pm \pi$ where the electron repulsion potential diverges. In the immediate vicinity of these genuine Fermi holes there always appear conjugate Fermi holes, but unlike in the former ones their spatial distribution strongly depends on the corresponding orbital configuration. In the cases of $(1 s)(2 p)$ and $\left(0 \sigma_{g}\right)\left(1 \pi_{u}\right)$ configurations the conjugate Fermi holes appear in regions defined by $r_{1}=r_{2}$ and $\phi_{-}= \pm \pi / 2$. The electron repulsion potential has also been visualised in the internal space which manifests itself as three striking poles peaked at $\phi_{-}=0, \pm \pi$.

The structure of genuine and conjugate Fermi holes and of the poles of the electron repulsion potential in the internal space explain the difference between the He-like and quantum dot systems: For the regime of large $Z_{\mathrm{n}}$ and $\omega$ the electron repulsion is sufficiently weak so that the internal wave functions of both the singlet and triplet states are close to their zeroth-order form that results when the electron repulsion is ignored. In this regime the singlet wave function has a large probability density in regions defined by the Fermi holes while the corresponding triplet wave function does not. Since the poles of the electron repulsion potential penetrate into the Fermi holes, the singlet state is characterized by a larger electron repulsion than the triplet thus supporting the traditional interpretation of Hund's multiplicity rule in the regime of large confinement.

However, as $Z_{\mathrm{n}}$ and $\omega$ decrease the electron repulsion potential becomes stronger at its poles and pushes the singlet probability density out of the Fermi holes. Since the electron repulsion potential affects the singlet internal wave function more strongly than the corresponding triplet wave function the one-body electron density distribution in the singlet and triplet states which are identical in the limit of large confinement become increasingly distinct. This explains the reason why for decreasing $Z_{\mathrm{n}}$ and $\omega$ the one-electron contribution to the singlet-triplet energy gap $\Delta E_{\text {one }}$ becomes rather large relative to the two-electron contribution $\Delta E_{\text {two }}$. The fate of the singlet probability density that migrated out of the vicinity of the Fermi holes then determines the distinct behavior in He-like and quantum dot systems in the regime of small $Z_{\mathrm{n}}$ and $\omega$. In the case of He-like systems the singlet probability density migrates towards the regions of either large $r_{1}$ or large $r_{2}$ while in the case of quantum dots it stays around the poles of the electron repulsion potential. This difference in the spatial distribution of the migrated singlet probability density in these systems thus originates from the difference in the shell structure of the Coulomb-type and harmonic-oscillator-type orbitals: The 
exponents of the one-electron orbitals in different principal shells $n$ of He-like systems significantly differ due to the cusp at the origin and the long tail of the Coulomb potential. On the other hand, the orbital exponents for quantum dots are identical for any polyad quantum number $v_{p}$ that specifies the shell structure of the harmonicoscillator potentials. Thus, in the case of He-like systems the products of these oneelectron orbitals, $\psi_{1 s}\left(\overrightarrow{r_{1}}\right) \psi_{2 p}\left(\overrightarrow{r_{2}}\right)$ and $\psi_{2 p}\left(\overrightarrow{r_{1}}\right) \psi_{1 s}\left(\overrightarrow{r_{2}}\right)$, which constitute the orbital part of a single-determinant wave function, are predominantly located along the $r_{1}$ and $r_{2}$ axes, respectively, due to the combination of a narrow $1 s$ and a broad $2 p$ orbital. Consequently, the singlet probability density that migrated out of the original region in the vicinity of strong potential poles had to move along the $r_{1}$ or the $r_{2}$ axes. Further, due to the presence of conjugate Fermi holes in regions around the Fermi holes, the singlet probability density has to migrate further away from the origin towards regions with large $r_{1}$ or large $r_{2}$. This results in a smaller electron repulsion and a smaller decrease of the nuclear attraction energy for the singlet state relative to the triplet state.

Finally, in the case of quantum dots the products of the one-electron orbitals $\psi_{0 \sigma_{g}}\left(\overrightarrow{r_{1}}\right) \psi_{1 \pi_{u}}\left(\overrightarrow{r_{2}}\right)$ and $\psi_{1 \pi_{u}}\left(\overrightarrow{r_{1}}\right) \psi_{0 \sigma_{g}}\left(\overrightarrow{r_{2}}\right)$ have a cylindrical distribution due to sharing the same orbital exponent. Therefore, plenty of space is available for the singlet probability density located originally in the area of the Fermi holes in order to avoid the poles of the electron repulsion potential. Unlike as in the He-like systems, the singlet probability that migrates out of the Fermi holes remains close to them simply avoiding the diverging points of the electron repulsion potential. This results in a larger electron repulsion of the singlet state relative to the triplet state even in the regime of small $\omega$.

\section{Aknowledgements}

The present study has been supported in parts by Grants-in-Aid for Scientific Research (No 23550025) from the Ministry of Education, Culture, Sports, Science and Technology (MEXT) and by Nihon University Strategic Projects for Academic Research. Two of us (T.S. and J.P.) would like to thank the Alexander von Humboldt Foundation for its kind support and Geerd H. F. Diercksen and the Max-Planck Institute for Astrophysics in Garching for their hospitality.

\section{References}

[1] Hund F 1925 Z. Phys. 33345

[2] Hund F 1925 Z. Phys. 34296

[3] Hund F 1927 Linienspektren und periodisches System der Elemente (Springer, Berlin) pp. 98, 124

[4] Rechenberg H 1996 in Hundert Jahre Friedrich Hund edited by M. Schroeder (VandenHoeck and Ruprecht, Goettingen), Nachrichten der Akademie der Wissenschaften in Göttingen. II Mathematisch-Physikalische Klasse pp. 4-32

[5] Herzberg G 1944 Atomic Spectra and Atomic Structure (Dover, New York) p. 135

[6] Szasz L 1992 The Electronic Structure of Atoms (Wiley, New York) p. 52

[7] Shore B W and Menzel D H 1968 Principles of Atomic Spectra (Wiley, New York) p. 101

[8] Friedrich H 2006 Theoretical Atomic Physics (Springer, Berlin) p. 105 
[9] Slater J C 1929 Phys. Rev. 34 1293-1322

[10] Davidson E R 1964 J. Chem. Phys. 41 656-658

[11] Davidson E R 1965 J. Chem. Phys. 42 4199-4200

[12] Messmer R P and Birss F W 1969 J. Phys. Chem. 73 2085-2086

[13] Kohl D A 1972 J. Chem. Phys. 56 4236-4238

[14] Katriel J 1972 Phys. Rev. A 5 1990-1992

[15] Katriel J 1972 Theoret. Chim. Acta 23 309-315

[16] Katriel J 1972 Theoret. Chim. Acta 26 163-170

[17] Katriel J and Pauncz R 1977 Adv. Quantum Chem. 10 143-185

[18] Boyd R J and Coulson C A 1973 J. Phys. B: At. Mol. Opt. Phys. 6 782-793

[19] Boyd R J and Coulson C A 1974 J. Phys. B: At. Mol. Opt. Phys. 7 1805-1816

[20] Boyd R J 1984 Nature 310 480-481

[21] Colpa J P, Thakkar A J, Smith Jr V H, and Randle P 1975 Mol. Phys. 29 1861-1875

[22] Regier P E and Thakkar A J 1984 J. Phys. B: At. Mol. Opt. Phys. 17 3391-3403

[23] Koga T, Matsuyama H, Dehesa J S, and Thakkar A J 1999 J. Chem. Phys. 110 5763-5771

[24] Shim I and Dahl J P 1978 Theor. Chim. Acta 48 165-174

[25] Hongo K, Maezono R, Kawazoe Y, Yasuhara H, Towler M D, and Needs R J 2004 J. Chem. Phys. $1217144-7147$

[26] Oyamada T, Hongo K, Kawazoe Y, and Yasuhara H 2006 J. Chem. Phys. 125014101

[27] Oyamada T, Hongo K, Kawazoe Y, and Yasuhara H 2010 J. Chem. Phys. 133164113

[28] Kutzelnigg W and Morgan III J D 1996 Z. Phys. D 36 197-214

[29] Colpa J P and Brown R E 1973 Mol. Phys. 26 1453-1463

[30] Sajeev Y, Sindelka M, and Moiseyev N 2008 J. Chem. Phys. 128061101

[31] Sako T, Paldus J, Ichimura A, and Diercksen G H F 2011 Phys. Rev. A 83032511

[32] Sako T, Paldus J, and Diercksen G H F 2010 Phys. Rev. A 81022501

[33] Johnson N F 1995 J. Phys.: Condens. Matter 7 965-989

[34] Sako T and Diercksen G H F 2007 Phys. Rev. B 75115413

[35] Sako T and Diercksen G H F 2008 J. Phys.: Condens. Matt. 20155202

[36] Sako T and Diercksen G H F 2003 J. Phys. B: At. Mol. Opt. Phys. 36 1433-1457

[37] Sako T and Diercksen G H F 2003 J. Phys. B: At. Mol. Opt. Phys. 36 1681-1702

[38] Sako T and Diercksen G H F 2003 J. Phys.: Condens. Matt. 15 5487-5509

[39] Sako T and Diercksen G H F 2005 J. Phys.: Condens. Matt. 17 5159-5178

[40] Sako T, Hervieux P A, and Diercksen G H F 2006 Phys. Rev. B 74045329

[41] Reeves C M 1963 J. Chem. Phys. 39 1-10

[42] Reeves C M and Harrison M C 1963 J. Chem. Phys. 39 11-17

[43] Wilson S 1984 Electron Correlation in Molecules (Oxford: Clarendon) pp. 180, 181

[44] Katriel J 2011 private communication

[45] Sako T, Paldus J, and Diercksen G H F 2009 Adv. Quantum Chem. 58 177-201

[46] Kellman E and Lynch E D 1986 J. Chem. Phys. 85 7216-7223 